\title{
Hydration dynamics of protein molecules in aqueous solution: Unity among diversity ${ }^{\#}$
}

\author{
BIMAN JANA $^{\mathrm{a}}$, SUBRATA PAL ${ }^{\mathrm{b}}$ and BIMAN BAGCHI ${ }^{\mathrm{a}, \mathrm{c}, *}$ \\ ${ }^{a}$ Solid State and Structural Chemistry Unit, Indian Institute of Science, Bangalore 560 012, India \\ b Indian Institute of Technology, Gandhinagar, Ahmedabad 382 424, India \\ ${ }^{\mathrm{c}}$ Jawaharlal Nehru Centre for Advanced Research, Jakkur, Bangalore 560 064, India \\ e-mail: bbagchi@sscu.iisc.ernet.in
}

\begin{abstract}
Dielectric dispersion and NMRD experiments have revealed that a significant fraction of water molecules in the hydration shell of various proteins do not exhibit any slowing down of dynamics. This is usually attributed to the presence of the hydrophobic residues (HBR) on the surface, although HBRs alone cannot account for the large amplitude of the fast component. Solvation dynamics experiments and also computer simulation studies, on the other hand, repeatedly observed the presence of a non-negligible slow component. Here we show, by considering three well-known proteins (lysozyme, myoglobin and adelynate kinase), that the fast component arises partly from the response of those water molecules that are hydrogen bonded with the backbone oxygen (BBO) atoms. These are structurally and energetically less stable than those with the side chain oxygen (SCO) atoms. In addition, the electrostatic interaction energy distribution (EIED) of individual water molecules (hydrogen bonded to SCO) with side chain oxygen atoms shows a surprising two peak character with the lower energy peak almost coincident with the energy distribution of water hydrogen bonded to backbone oxygen atoms (BBO). This two peak contribution appears to be quite general as we find it for lysozyme, myoglobin and adenylate kinase (ADK). The sharp peak of EIED at small energy (at less than $\left.2 k_{\mathrm{B}} T\right)$ for the $B B O$ atoms, together with the first peak of EIED of SCO and the HBRs on the protein surface, explain why a large fraction $(\sim 80 \%)$ of water in the protein hydration layer remains almost as mobile as bulk water. Significant slowness arises only from the hydrogen bonds that populate the second peak of EIED at larger energy (at about $4 \mathrm{k}_{\mathrm{B}} \mathrm{T}$ ). Thus, if we consider hydrogen bond interaction alone, only 15-20\% of water molecules in the protein hydration layer can exhibit slow dynamics, resulting in an average relaxation time of about 5-10 ps. The latter estimate assumes a time constant of $20-100$ ps for the slow component. Interestingly, relaxation of water molecules hydrogen bonded to back bone oxygen exhibit an initial component faster than the bulk, suggesting that hydrogen bonding of these water molecules remains frustrated. This explanation of the heterogeneous and non-exponential dynamics of water in the hydration layer is quantitatively consistent with all the available experimental results, and provides unification among diverse features.
\end{abstract}

Keywords. Protein-water hydrogen bond; electrostatic energy distribution; faster than bulk.

\section{Introduction}

Dynamics of water in the hydration shell of proteins in aqueous solution is heterogeneous, and the decay of relevant time correlation functions is non-exponential. In this article we present an explanation of the different time scales that have been observed in different experiments and provide a unified description, by considering three well-known proteins, namely, lysozyme, myoglobin and adelynate kinase (ADK). We show that there is a certain degree of universality or commonality in the behaviour of hydration shells across the proteins,

\footnotetext{
\#Dedicated to Prof. N Sathyamurthy on his 60th birthday

*For correspondence
}

even when the surface that bulk water faces varies from protein to protein on a microscopic length scale.

While the study of the structure and dynamics of hydrogen bonds of water with solutes has a long history, ${ }^{1}$ increased attention on the dynamics of water in protein hydration layer is a more recent phenomenon. ${ }^{2-13}$ The rate of the breaking of hydrogen bond between any two water molecules within the layer is found to be, on an average, slower than those in the bulk water. The extent of the slowness has been a subject of great debate, with quoted time scales often differing by more than two orders of magnitude. However, it is hard to uniquely characterize the lifetime of hydrogen bonds within the hydration layer around a protein as the protein offers a complex and heterogeneous surface. ${ }^{13-18}$ Therefore, one should really think in terms of a distribution of lifetimes and average properties 
should be computed over this distribution, although in many biological applications, hydrogen bond dynamics near a given region of exposed protein surface is relevant.

In spite of severe disruption of the hydrogen bond by the protein surface, water can still form a quasi twodimensional connected hydrogen bond network/cluster around the protein. ${ }^{19,20}$ This two-dimensional network itself is constrained due to its interaction with the protein hydrophilic and hydrophobic groups. However, due to the heterogeneous chemical nature of the protein surface, the network will necessarily have different rigidity in different regions of the surface.

In understanding the dynamics of the hydration shell, we need to make a distinction between two distinct parts of the protein, namely the backbone and the side chain, that play critical role in determining its folded 3 -dimensional structural topology. ${ }^{20-22}$ On an average, $60 \%$ of the surface polar atoms belong to the backbone chain, consisting of polar amide groups. In the folded state, the backbone atoms form a rigid framework, while the side chains remain mobile to provide flexibility to the structure. Recent studies have revealed the importance of the flexibility of the side chains of protein in the dynamics of the hydration layer. ${ }^{16-18}$ However, the structure and dynamics of the hydrogen bonds between the water molecule and between water and protein are all influenced by the extended hydrogen bonded network that surrounds the protein. From the protein's perspective, the water molecules which are in direct contact with protein atoms form a rugged twodimensional network. This group of inter-connected surface water molecules is expected to behave differently from the bulk because of the interaction with the protein groups. The ruggedness of this quasitwo-dimensional network is determined both by the topology and the polarity of the protein groups. Therefore, both the stability and the mobility of this extended hydrogen bond network depend on the protein-water $\mathrm{H}$-bond lifetime dynamics, in addition to the interaction with the bulk water. All these interactions are responsible for the dynamical behaviour of the protein hydration layer and also of the time scale of motion of the side chain atoms. These aspects have not yet been studied in quantitative detail.

Despite the diversity of protein-water hydrogen bond and interactions at the hydration layer, one can still search for certain general features which can help systematize and simplify the complexity of structure and dynamics. One such general result that has been observed and discussed in detail is the difference in water structure and dynamics around hydrophobic and hydrophilic groups. Both structure and dynamics of water around the hydrophobic groups are substantially different from those around hydrophilic groups. Since the hydrophilic groups at the protein surface can again be divided into groups belonging to the side chain and the backbone, one can look into further systematization in terms of this difference. Such a study is the objective of the present work. We should mention that such a distinctive study involving dynamics near the backbone atoms has not been presented before. We demonstrate below, such a study helps in understanding the time scales of the hydration layer.

The initial measurement of dielectric relaxation of water in aqueous protein solution observed an additional dispersion (often termed as $\delta$-dispersion) in the gigahertz (GHZ) region. ${ }^{1-7}$ Subsequently, Nandi and Bagchi proposed a dynamic exchange model where kinetics of protein-water hydrogen bond breaking was discussed as an activated process that contributes to slow dynamics in the hydration layer. ${ }^{8,9}$ Several experimental studies have confirmed ${ }^{23,24}$ or disputed ${ }^{25}$ the dynamic exchange model. Simulation study by Pizzitutti et al. have revealed that the structure and dynamics of the protein hydration layer is primarily determined by the interaction of the water molecules with the protein. ${ }^{16}$ In a recent study by Murarka et al. have discovered the role of hydrophobic residues in this context ${ }^{14,15}$ and Jana et al. found that the mechanism of hydrogen bond breaking is different in the hydration layer from that in the bulk. ${ }^{26}$ While in the bulk, the H-bond breaking mechanism involves both the orientational and the translational motion of the two water molecules involved in the $\mathrm{H}$-bond exchange, it is mainly the orientational motion that leads to H-bond exchange in the hydration layer. That is, the 5th neighbour does not move by any substantial distance. This indicates the localization of the water molecules in a two-dimensional network.

The extent of slow dynamics of water in the hydration layer has been a subject of great controversy in recent times. Solvation dynamics experiments have suggested the presence of time scales in the range of $20-100 \mathrm{ps}$, and sometimes even longer. This is to be contrasted with solvation in bulk water where slowest time scale is of the order of only about 1 ps. Magnetic resonance experiments have suggested time scales only 2-3 times longer than bulk water. Computer simulations have revealed time scales that seem to agree with solvation dynamics experiments. However, it should be noted that solvation dynamics experiments give estimate of the slow time scales while NMR experiments measure the average time. So, there might not be any great 
discrepancy there. However, no study has yet provided a satisfactory quantitative resolution of the difference. Such a resolution is provided here.

In this article, we analyse the structure and energetics of the hydrogen bonds involving the oxygen atom of the backbone and side chains and their role in determining the lifetime dynamics of protein-water hydrogen bonds. We then discuss about the mobility of water molecules around the polar groups of protein along with a discussion on the correlation of the same with dynamics of hydrogen bond breaking.

The main result of this study is that the electrostatic interaction energy distribution (EIED) of hydrogen bonds of water with side chain oxygen atoms shows a surprising two peak character with the lower energy peak nearly coincident with the energy distribution of hydrogen bonds involving backbone oxygen atoms. The peak of EIED at low energy corresponds to weak hydrogen bonds which belong to water molecules which exhibit faster dynamics. Since $60 \%$ of the surface atoms belong to the backbone, implies that many, if not most, water molecules in the layer are as faster as the bulk water in terms of H-bond dynamics. There are, however, still $15-20 \%$ of hydrogen bonds which are strong and water molecules forming these bonds are slower in their rotational and translational dynamics. Second important finding is that if we quench the motion of the side chain atoms, the decay of hydrogen bond time correlation function becomes faster at long times, although a bit slower at short times. This clearly indicates the participation of the motion of protein side chains in hydrogen bond lifetime dynamics. This is physically easy to understand because the coupled motion of protein side chain and water molecules that are hydrogen bonded to it will strive to stay H-bonded if the bonding is strong.

The organization of the rest of the paper is as follows. In section 2, we discuss about the methods and details of the simulation used in this study. Section 3 discusses about the pattern of protein-water hydrogen bonds. In section 4, we present the dynamical analysis of proteinwater hydrogen bonds along with mobility analysis of water molecules. Conclusions are given in section 5 .

\section{Methods}

\subsection{Protein lysozyme}

Lysozyme is a globular protein with 129 amino acids, of which 69 amino acids are hydrophilic and other 60 amino acids are hydrophobic. In its solvated state, many of the water molecules in the hydration layer are $\mathrm{H}$ bonded to the polar groups of the amino acid residues and the backbone. At the same time, many more of the water molecules in the hydration layer are not hydrogen bonded to the protein especially when they are near the hydrophobic residues. On an average, approximately $30 \%$ of the total water molecules in the hydration layer of lysozyme are H-bonded to protein (with the polar group of backbone and side-chains).

\subsection{Molecular dynamics simulation}

We have carried out classical molecular dynamics simulations using the well-known GROMACS package. ${ }^{27}$ We have used SPC-E model for water molecules. ${ }^{28}$ The trajectory of the bulk water was propagated with a leapfrog integrator with a time step of $0.5 \mathrm{fs}$. We have employed the periodic boundary condition, and the long-range Coulombic interactions were calculated using Ewald summation. ${ }^{29}$ The bulk water system (481 water molecules) was equilibrated for 50 -ps at $300 \mathrm{~K}$ in canonical ensemble and then the trajectory was propagated in a microcanonical ensemble for $100 \mathrm{ps}$. For the lysozyme-water system (lysozyme in $5995 \mathrm{SPC} / \mathrm{E}$ water), we have equilibrated the system in a NPT ensemble at $300 \mathrm{~K}$ and the trajectory was propagated in a NVT ensemble.

\subsection{Definition of $\mathrm{OH} \ldots \mathrm{O}$ and $\mathrm{OH} \ldots \mathrm{OP} \mathrm{H}$-bond}

To detect the H-bond switching events along a molecular dynamics trajectory, criteria for the existence of the $\mathrm{H}$-bond have to be chosen. We have chosen the widely used geometric definition: ${ }^{30} \mathrm{r}_{\mathrm{OO}}<3.5 \AA, \theta_{\mathrm{HOO}}<30^{\circ}$, where $\mathrm{r}_{\mathrm{OO}}$ is the distance between the donor and the acceptor oxygen atoms, and $\theta_{\mathrm{HOO}}$ is the angle between the $\mathrm{O}-\mathrm{H}$ bond and the $\mathrm{OO}$ vectors for the waterwater hydrogen bonds in bulk water. For protein-water hydrogen bonds, we have used the geometric definition: ${ }^{31,32} \mathrm{r}_{\mathrm{OO}}<3.5 \AA, \theta_{\mathrm{OH} \ldots \mathrm{O}}>140^{\circ}$, where $\theta_{\mathrm{OH} \ldots \mathrm{O}}$ is the angle between the $\mathrm{O}-\mathrm{H}$ bond and $\mathrm{H}$... O hydrogen bond vectors.

\section{Hydrogen bond pattern}

\subsection{Classification of protein-water hydrogen bonds}

We first categorized the protein-water hydrogen bonds into two types. The hydrogen bond involving backbone oxygen atom is designated as BBO-W and that involving side chain oxygen atom is designated as SCO-W. The bulk water hydrogen bond is referred as BWO-W here after. 


\subsection{Energy distribution of hydrogen bonds}

We now discuss the structure and energetics of the two types of protein-water bonds. We first concentrate on the energetics of the BBO-W and SCO-W hydrogen bonds. We have calculated the electrostatic energy between the oxygen atom of the backbone or the side chain and the water molecule which is hydrogen bonded to that oxygen atom. The origin of the stabilization of the hydrogen bonds is mostly electrostatic. ${ }^{16}$ The distributions of the electrostatic energy of the BBO-W and SCO-W bonds are displayed in figure 1a. While the distribution for SCO-W bonds is found to have a two peak structure (fitted to two Gaussian distributions), the distribution of electrostatic energy for BBO-W bonds is single peaked and is in the lower energy (less stable hydrogen bond) region. Interestingly, the lower energy peak position of SCO-W and the peak position of $\mathrm{BBO}-\mathrm{W}$ are found to be same. It is clearly evident from the energy distributions that in general SCO-W bonds are more stable energetically than BBO-W hydrogen bonds due to the presence of a higher energy (more stable hydrogen bond) peak in the distribution for the former.

We have also calculated the electrostatic energy distribution of SCO-W and BBO-W hydrogen bonds in myoglobin and adenylate kinase. The distributions are shown in figure $1 \mathrm{~b}, \mathrm{c}$. We find a similar two peak contribution (fitted to two Gaussian distributions) in the electrostatic energy distribution of SCO-W hydrogen bonds. This result implies that the two peak structure of the electrostatic energy distribution of the SCO-W hydrogen bonds in proteins is a general phenomenon.

The lower energy peak can be assigned to the hydrogen bonds where SCO is involved in intra-molecular hydrogen bonding with the protein hydrogen atoms to maintain its $3 \mathrm{D}$ structure. As the intra-molecular hydrogen bonds are generally found to be strong, the hydrogen bond with the water molecule is less stable. The higher energy peak can be assigned to the hydrogen bonds where SCO is not involved in intramolecular hydrogen bonding with the protein hydrogen atoms. On the other hand, backbone oxygen atoms are mostly involved in strong intra-molecular hydrogen bonds to maintain the rigid backbone structure of the protein and eventually the hydrogen bonding with water becomes weaker. Distance and angle distributions of these two types of H-bonds also support the average picture described above.

The recent study by Pizzitutti et al. has revealed that the water structure and dynamics near the protein surface depend on the surface topology and energetic disorder. ${ }^{16}$ They have also quantified the separate effects of both on the anomalous dynamics of hydration
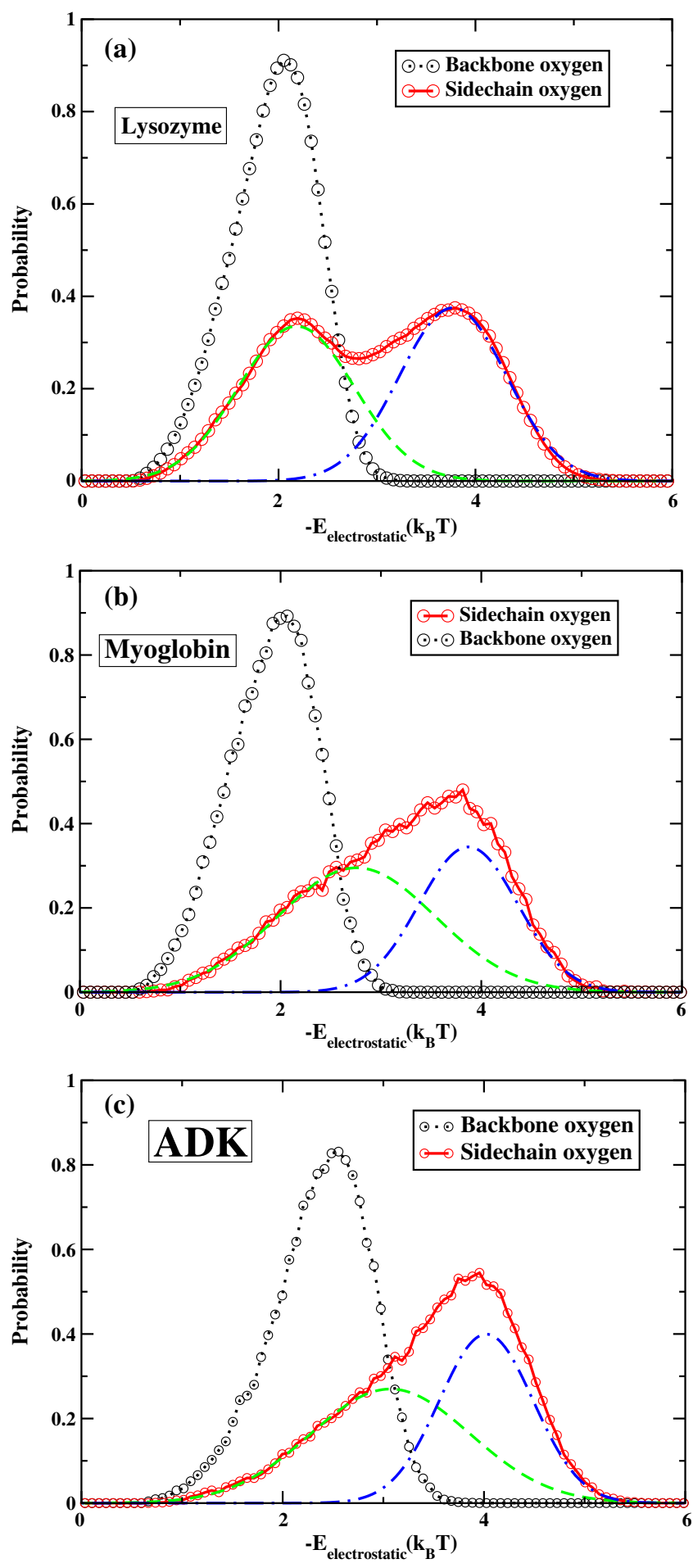

Figure 1. Energetics of protein-water hydrogen bonds. Distribution of electrostatic energy of water molecules (which are hydrogen bonded with $\mathrm{BBO}$ and SCO) with backbone and side chain oxygen atoms for (a) lysozyme, (b) myoglobin and (c) adenylate kinase. Note the bimodal character of the distribution for side chain oxygen atoms. See text for further discussion.

layer water. Interestingly, they found that the reorientation dynamics primarily depends on the energetic disorder only and indeed found that the water molecules 
in the hydration layer reorient faster than in the bulk when the electrostatic interaction between protein and water is switched off. ${ }^{16}$ In a classic study, Cheng and Rossky had earlier demonstrated that the structure of water molecules around hydrophobic groups of proteins largely depends on surface topology. ${ }^{33}$ Two distinct structures are observed near a biomolecular surface which can be distinguished by a substantial difference in the water-water enthalpy. While clathrate-like structures dominate near the convex surface, the hydration shell near flat surfaces fluctuates between clathratelike and less-ordered inverted structures. ${ }^{33}$ In a separate study, they have also discussed the effect of vicinal polar and charged group on hydrophobic hydration. ${ }^{34}$

The electrostatic interaction energy in the first peak is smaller than or comparable to that of bulk water. Hence, these water molecules can rotate even faster than bulk water. ${ }^{16}$ However, ones in the second peak can be slow by one to two orders of magnitude. ${ }^{9}$

\section{Analysis of hydrogen bond breaking dynamics}

We now focus on the dynamics of the different proteinwater hydrogen bonds (BBO-W and SCO-W) and compare the same with bulk water hydrogen bonds (BWO-W). The dynamics have been explored as following.

\subsection{Rate of hydrogen bond breaking}

We have calculated the forward rate of hydrogen bond breaking for different kinds of hydrogen bonds (BWO$\mathrm{W}, \mathrm{BBO}-\mathrm{W}$, and SCO-W) considered in the present work. To this end, we have calculated the correlation function $\left(1-\left\langle n_{a}(0) n_{b}(t)\right\rangle\right)$, where $n_{a}$ is 1 if a particular pair is hydrogen bonded and 0 otherwise, and $n_{b}$ is 1 when that particular pair is broken and 0 otherwise. ${ }^{26,35}$ Transient bond breaking has been neglected. In addition, the absorbing boundary condition is used for $\mathrm{n}_{b}$ so that when a particular hydrogen bond has been broken, it never reforms. We thus discard any contribution from back reaction and the rate constant extracted from these correlation functions will give the forward rate of hydrogen bond switching of a particular pair. Figure $2 \mathrm{a}$ shows the decay of the correlation functions for different pairs in a semi-log plot. The decay of the correlation function for BWO-W bonds is mono-exponential with a characteristic time constant $\tau_{0} \sim 1.8 \mathrm{ps}$ and this estimate is in agreement with earlier simulation studies. ${ }^{35}$ The decays of the correlation function of BBO-W and SCO-W bonds are bi-exponential with characteristic average time $\left(<\tau_{0}>\right) 1.58 \mathrm{ps}$ and $5.37 \mathrm{ps}$, respectively.
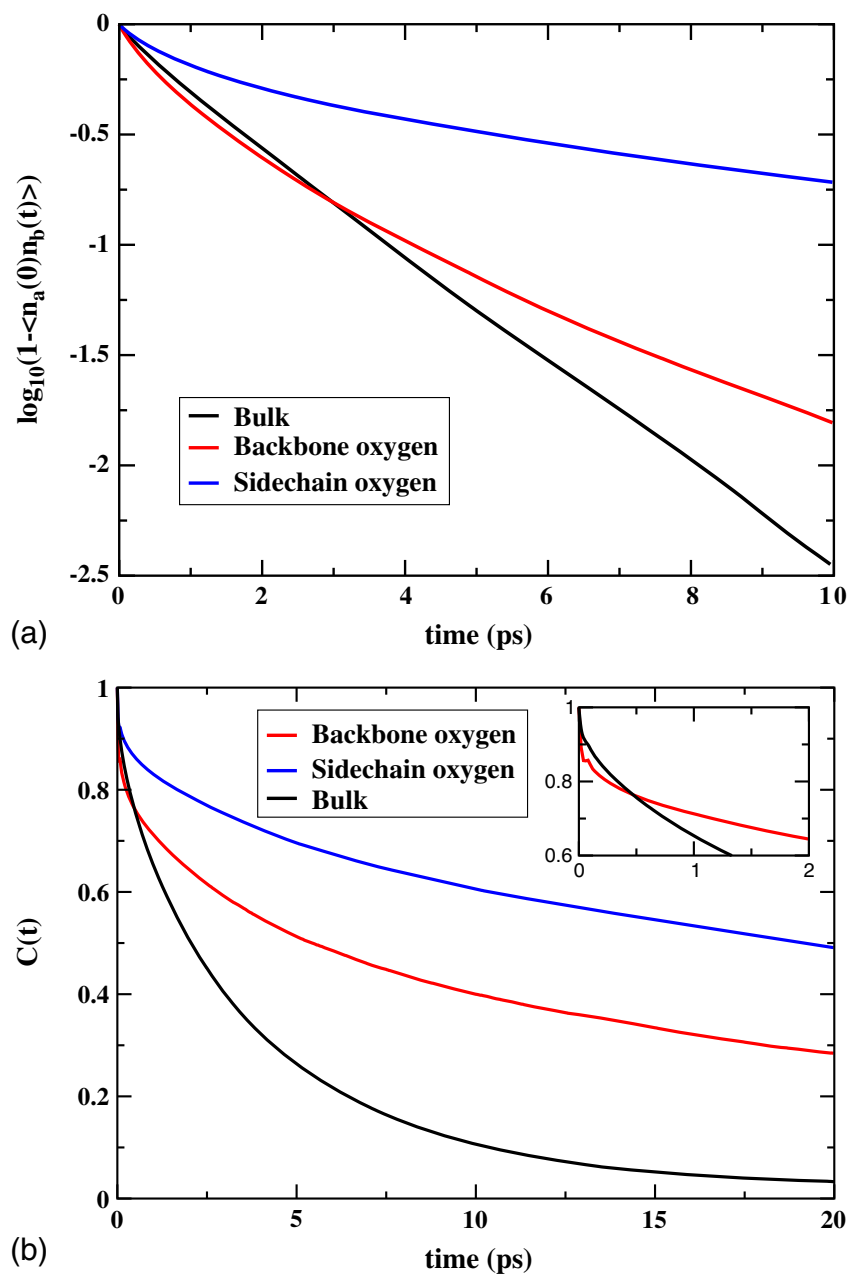

Figure 2. (a) Decay of the H-bond correlation function in a semi-log plot for BBO-W, SCO-W, and in bulk water. Slope of this correlation function provides information about the $\mathrm{H}$ bond switching rate. Note the slow decay of the correlation function for SCO-W. Note also the crossover between the correlation functions for bulk water and BBO-W. (b) Decay of $C(t)$ function for BBO-W, SCO-W and in bulk water. Inset of the $C(t)$ plot shows the crossover between $C(t)$ functions for BBO-W and in bulk water at intermediate time.

From the plot and the characteristic average time values calculated from the fitting parameters, it is clearly evident that the rate of hydrogen bond breaking of SCO$\mathrm{W}$ bonds is slower than of $\mathrm{BBO}-\mathrm{W}$ bond which in turn has a faster breaking rate than BWO-W bonds. Note that the trend of hydrogen bond breaking rate between BBO-W and SCO-W bonds is well-correlated with the trend in structure and energetics between them.

\subsection{Hydrogen bond lifetime}

To explore the dynamics of the hydrogen bonds in a more conventional way, we have calculated hydrogen 
bond lifetime correlation functions $[S(t)$ and $C(t)]$ which are defined as ${ }^{36,37}$

$$
S(t)=\frac{\langle h(0) H(t)\rangle}{\langle h(0) h(0)\rangle} \quad \text { and } \quad C(t)=\frac{\langle h(0) h(t)\rangle}{\langle h(0) h(0)\rangle} .
$$

Here, $h(t)$ is considered to be unity if a particular pair of sites (BWO or $\mathrm{BBO}$ or $\mathrm{SCO}$ and $\mathrm{W}$ ) is hydrogen bonded at time $t$ according to the definition of the hydrogen bond, and zero otherwise. $H(t)$ is considered to be unity if a pair of sites is bonded continuously for the time interval 0 to $t$, and zero otherwise. The function $S(t)$ provides the information about the probability that a particular pair is hydrogen bonded continuously for the interval 0 to $t$ and the function $C(t)$ describes the probability that a particular pair is hydrogen bonded at time $t$, given that it was hydrogen bonded at $t=0$. The decay of $S(t)$ function shows that SCO-W has a larger lifetime than BWO-W which in turn has a larger lifetime than BBO-W (not shown here). The decay of $C(t)$ function shows that SCO-W bonds has a larger lifetime than BBO-W bonds which in turn has a larger lifetime than BWO-W bonds (as shown in figure 2b). Note that $C(t)$ function decays faster for $\mathrm{BBO}-\mathrm{W}$ bonds than BWO-W bonds initially and decay behaviour becomes reverse at longer time giving rise to a crossover at shortto-intermediate time (as shown in the inset of figure $2 b$ ). We have also noted that interestingly the behaviour of $C(t)$ and $S(t)$ function are exactly opposite for BBO$\mathrm{W}$ and BWO-W bonds. $S(t)$ of BBO-W bonds decays faster than BWO-W bonds which indicates that the strength of former is less than the latter. Whereas the decay of $C(t)$ of BBO-W bonds is slower than BWO$\mathrm{W}$ bonds which suggests that the probability of reformation of the former is more compared to the latter. We will discuss about this interesting behaviour from the view point of mobility of water molecules around the protein surface later.

\subsection{Water mobility around protein: A cluster analysis}

Due to complex heterogeneous structure and interaction present on the protein surface, the dynamics of water molecules can vary widely from place to place. Here we have investigated the mobility of water molecules around a central acceptor molecule [e.g., bulk water oxygen atom (BWO), backbone oxygen atom (BBO), and side-chain oxygen atom (SCO)] of the respective hydrogen bonded pairs. To this end, we have calculated a correlation function $\left(S_{\text {cluster }}(t)\right)$ which is defined as ${ }^{36}$

$$
S_{\text {cluster }}(t)=\frac{\langle n(0) N(t)\rangle}{\langle n(0) n(0)\rangle} .
$$

Here, $n(t)$ for a water molecule is considered to be unity if that water molecule is within $3.5 \AA$ from a particular central acceptor atom at time $t$, and zero otherwise. $N(t)$ for a water molecule is considered to be unity if that water molecule is within $3.5 \AA$ from a particular central acceptor atom continuously for time interval 0 to $t$, and zero otherwise. The function $S_{\text {cluster }}(t)$ provides information about the probability that a water molecule is within $3.5 \AA$ from a particular central acceptor atom continuously for time interval 0 to $t$. Figure 3 displays the decay of $S_{\text {cluster }}(t)$ for different central acceptor atoms. One can clearly observe from the decay of $S_{\text {cluster }}(t)$ that the water molecules around SCO is less mobile than those around BBO which is in turn less mobile than those around BWO. The decay pattern of the correlation functions (faster for bulk and slower for protein surface) has a direct consequence with the presence of a water cluster around protein surface with extensive connectivity. ${ }^{16-18}$ It can also be noted that the decay of $S_{\text {cluster }}(t)$ faster for BBO than BWO initially and decay behaviour becomes reverse at longer time giving rise to a crossover at short-to-intermediate time (as shown in insets).

We have also explored the correlation between the dynamical features observed and the mobility analysis presented above. Mobility analysis for the water molecules around $\mathrm{BBO}$ and $\mathrm{BWO}$ can explain the reverse behaviour observed in $C(t)$ and $S(t)$ function between BBO-W and BWO-W bonds. Due to the less mobility of the cluster around $\mathrm{BBO}$, the broken $\mathrm{BBO}-$ $\mathrm{W}$ bonds have more probability to reform and hence a slow decay of $C(t)$ is observed. On the other hand, due to energetics reason, the less strong $\mathrm{BBO}-\mathrm{W}$ bonds

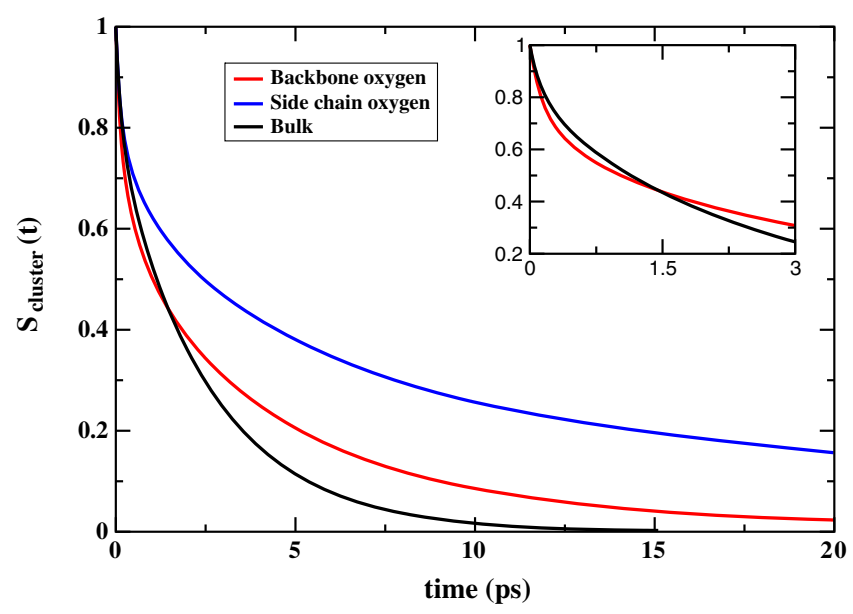

Figure 3. Mobility of water molecules around the polar group of protein. Decay of $S_{\text {Cluster }}(t)$ function for BBO, $\mathrm{SCO}$, and bulk water oxygen. Insets show the intermediate time crossover between the correlation function of $\mathrm{BBO}$ and bulk water oxygen. 
are bonded for less time interval constantly giving rise to a faster decay of $S(t)$ function. Also, the crossover observed for the decay of $C(t)$ function between BBO and BWO at short-to-intermediate time may have some correlation with the crossover observed in $S_{\text {cluster }}(t)$.

\subsection{Effect of protein side chain motion}

We now discuss about the role of protein side chain motion on the slow dynamics of water around the protein surface. Recent study by Pizzitutti et al. ${ }^{16}$ reveals that if the protein motion is constrained, the dynamics becomes more anomalous and slow. Another study by Li et al., ${ }^{16-18}$ showed that the slow long time component of solvation dynamics disappears when the protein is constrained. Figure 4 displays the $S(t)$ function in semilog plot for two different conditions, (i) when the protein motion is not constrained (natural) and (ii) when it is constrained (constrained), for SCO-W pair. $S(t)$ has a long time tail in its natural condition, the function initially decays slowly in its constrained condition compared to its natural condition and then decays to zero at long time giving rise to a crossover between the two $S(t)$ functions in the intermediate time. The disappearance of the long time tail for the constrained form is clearly evident from figure 4 . Thus, the slow component of the $S(t)$ function of SCO-W bonds is clearly due to the coupled motion between water and protein side chain.

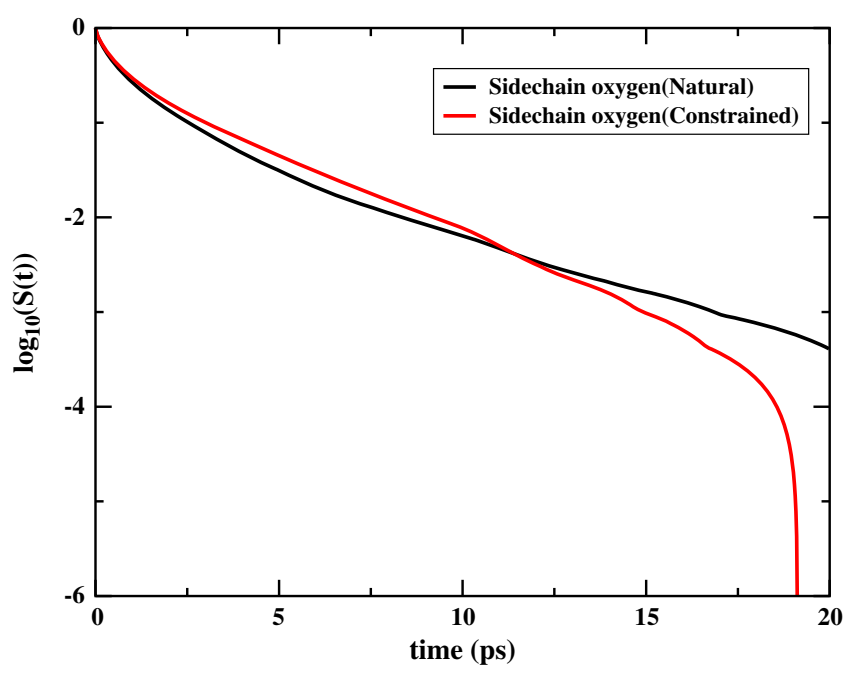

Figure 4. Effect of protein motion on the dynamics of hydrogen bonds. Decay of $S(t)$ function in semi-log plot for $\mathrm{SCO}-\mathrm{W}$ in natural and constrained protein motion conditions. Note the disappearance of the long time tail of the $S(t)$ function of SCO-W for the constrained protein motion condition.
Disappearance of long time tail in the hydrogen bond lifetime correlation function $S(t)$ and also in solvation time correlation function ${ }^{16-18}$ presents an intriguing problem. As noted in a recent study, water dynamics in general is expected to become slow and anomalous when the motion of protein side chain is quenched. ${ }^{16}$ This can be easily understood if we consider the effects of side chain interactions on water molecules. When the side chain motion is quenched, the force-force time correlation function giving rise to the frequency dependent friction develops a slow tail which gives rise to nonexponential dynamics in such observables as dynamic structure factor. However, the disappearance of long time tail in $S(t)$ seems to have a different explanation. When the motion of SCO is unconstrained, it can respond to the motion of water molecules that are bonded to it. This motion can retard breaking of strong hydrogen bonds. In certain sense, it is very much like barrier re-crossing dynamics one observes in chemical reaction dynamics, for example in Kramers' problem. Therefore, constrained SCO gives rise to faster $S(t)$ at longer times.

\subsection{Average relaxation time}

Experiments have revealed the relaxation of a slow time constant in the relaxation of the hydration layer. The estimate of this slow time constant varies from system to system. For proteins, the reported values of this slow time constant vary from 20 to $100 \mathrm{ps}$. According to the present analysis, only $15-20 \%$ of the hydration layer may contribute to this slow component. Rest may decay with time constant less than $1 \mathrm{ps}$. Therefore, the average time constant should range between 4 and 20 ps. In many cases, like lysozyme, the range may lie between 5 and 10 ps only. This estimate of average rate is in agreement with magnetic relaxation dispersion (MRD) experiments which also measures the average rate. However, present study also predicts that there can be a small (15-20\%) slow component of time scale $100 \mathrm{ps}$ or above. The slow component of time scale 100 ps has been observed in the experiments. ${ }^{5}$ This picture appears to be in agreement with most of the experimental results.

However, note that for some protein, like myoglobin, the number of polar groups is larger, implying the presence of significant slow component.

\subsection{Origin of the ultra-slow component}

Experiments using solvation dynamics and QENS at protein hydration layer often find a slow component of 
$100 \mathrm{ps}$ and above. In our present study, we find a component in the electrostatic energy distribution of water molecules with a weight of $15-20 \%$ that has binding energy above $4 \mathrm{k}_{\mathrm{B}} \mathrm{T}$. The diffusion coefficient of such water molecules will be reduced by approximately two orders of magnitude. Thus, a small component (15$20 \%$ ) in the relaxation of the protein hydration layer can indeed be of the order of $100 \mathrm{ps}$.

There are two more sources by which ultra-slow component may arise in the relaxation of the protein hydration layer.

(i) The probe used in many solvation dynamics experiments can slowly move away from the surface of the protein to the bulk. This slow translational diffusion of the probe can give rise to a ultra-slow component of the solvation dynamics. This issue has already been discussed earlier. ${ }^{1,5}$

(ii) Another source of the slow component can be conformational fluctuation of the protein. Example of such a protein is adenylate kinase (ADK) which can fluctuate between its open and closed form. This can give rise to a slow component in the solvation dynamics even of the order of nanosecond and slower.

\section{Conclusions}

We have analysed the dynamics of the hydrogen bond between water molecules and protein oxygen atoms in aqueous solution of lysozyme, myoglobin and adelynate kinase (ADK). We find that the hydrogen bond breaking dynamics are different for SCO-W from BBO-W. The former exhibits much slower bond breaking dynamics. From the bimodal character of the protein-water H-bond energy distribution observed for the side chain oxygen atoms, one can infer that there are two different types of $\mathrm{H}$-bond acceptor (oxygen atoms) present in the side chain. The stronger H-bonds are those with charged groups Asp and Glu, while the weaker bonds are with the rest. Energy distribution (figure 2) suggests that the energy of $\mathrm{H}$-bond of the weaker SCO-W bonds is similar to the BBO-W bonds. These bonds are therefore expected to show faster dynamics while the stronger SCO-W bonds are expected to exhibit slower dynamics and responsible for the slow long time dynamics.

The energy distribution also indicates that the hydration layer of the protein contains many fast water molecules along with a few slow water molecules. Since $50 \%$ of the surface groups are typically hydrophobic and also approximately $60-70 \%$ of the oxygen containing hydrophilic groups form weak $\mathrm{H}-$ bonds, only about $15-20 \%$ of the H-bonds at the surface of a protein molecule are strong and can exhibit slow dynamics. This is in agreement with the observation that the slow component has only a $15-20 \%$ weight in the total relaxation, while the rest exhibit faster dynamics. The dynamics of these strong hydrogen bonds may be described by a dynamic exchange model described earlier. However, the bond breaking dynamics is expected to be quite involved because of the participation of side chain motion and of other water molecules. This issue needs further study.

Finally, we stress that we have considered three wellknown proteins, namely, lysozyme, myoglobin and adelynate kinase (ADK) and found that there is certain degree of universality or commonality in the behaviour of hydration shells across the proteins, even when the surface that bulk water faces, varies from protein to protein on a microscopic length scale. Thus, there is a certain degree of unity among diversity which presents a rather satisfying scenario in this difficult problem.

\section{Acknowledgments}

This work has been supported partly by the grants from The Department of Science and Technology (DST) and JC Bose Fellowship, India. We thank Dr. S Roychowdhury and Mr. B V Adkar for helpful discussions.

\section{References}

1. (a) Bagchi B 2005 Chem. Rev. 105 3197; (b) Nandi N, Bhattacharyya K and Bagchi B 2000 Chem. Rev. 100 2013

2. Levy Y and Onuchic J N 2006 Annu. Rev. Biophys. Biomol. Struct. 35389

3. Raschke T M 2006 Curr. Opin. Struct. Biol. 16152

4. Ohmine I and Tanaka H 1993 Chem. Rev. 932545

5. (a) Bhattacharyya K 2008 Chem. Commun. 2848; (b) Bhattacharyya K 2003 Acc. Chem. Res. 36 95; (c) Sahu K, Mondal S K, Ghosh S and Bhattacharyya K 2007 Bull. Chem. Soc. Jpn 801033

6. Daniel R M, Finney J L and Stoneham M 2004 Philos. Trans. R. Soc. London Ser. B 3591143

7. Timasheff S N 1993 Annu. Rev. Biophys. Biomol. Struct. 2267

8. Nandi N and Bagchi B 1997 J. Phys. Chem. B 10110954

9. Nandi N and Bagchi B 1998 J. Phys. Chem. A 1028217

10. Chakraborty S and Bandopadhyay S 2007 J. Phys. Chem. B 1117626

11. Chakraborty S, Bandopadhyay S and Bagchi B $2005 \mathrm{~J}$. Am. Chem. Soc. 12716660

12. Pal S, Maiti P K and Bagchi B 2006 J. Chem. Phys. 125 234903

13. Jordanides $X$ J, Lang $M J$, Song $X$ and Fleming $G R$ 1999 J. Phys. Chem. B 1037995

14. Murarka R K and Head-Gordon T 2008 J. Phys. Chem. B 112179 
15. Murarka R K and Head-Gordon T 2007 J. Chem. Phys. 126215101

16. Pizzitutti F, Marchi M, Sterpone F and Rossky P J 2007 J. Phys. Chem. B 1117584

17. Hassanali A A, Li T, Zhong D and Singer S J 2006 J. Phys. Chem. B 11010497

18. Li T, Hassanali A A, Kao Y-T, Zhong D and Singer S J 2007 J. Am. Chem. Soc. 1293376

19. Khoshtariya D E, Hansen E, Leecharoen R and Walker G C 2003 J. Mol. Liq. 10513

20. Yokomizo T, Higo J and Nakasako M 2005 Chem. Phys. Lett. 41031

21. Thibodeaul $\mathrm{P} \mathrm{H}$, Brautigam $\mathrm{C}$ A, Machius $\mathrm{M}$ and Thomas P J 2004 Nat. Struct. Mol. Biol. 1210

22. Honig B and Cohen F E 1996 Folding Design 1 R17

23. Sen P, Mukherjee S, Halder A and Bhattacharyya K 2004 Chem. Phys. Lett. 385357

24. Pal S K, Peon J, Bagchi B and Zewail A H 2002 J. Phys. Chem. B 10612376

25. (a) Halle B 2004 Phil. Trans. R. Soc. Lond. B 359 1207; (b) Halle B and Davidovic M 2003 Proc. Natl. Acad. Sci. USA 10012135
26. Jana B, Pal S and Bagchi B 2008 J. Phys. Chem. B 112 9112

27. van der Spoel B, Lindahl E, Hess B, van Buuren A R, Apol E, Meulenhoff P J, Tieleman D P, Sijbers A L T M, Feenstra K A, van Drunen R and Berendsen H J C 2004 Gromacs User Manual version 3.2, www.gromacs.org

28. Berendsen H J C, Grigera J R and Straatsma T P 1987 J. Chem. Phys. 916269

29. (a) Darden T, York D and Pedersen L 1993 J. Chem. Phys. 98 10089; (b) Essmann U, Perera L, Berkowitz M L, Darden T, Lee H and Pedersen L G 1995 J. Chem. Phys. 1038577

30. Rey R, Møller K B and Hynes J T 2002 J. Phys. Chem. A 10611993

31. Schneider B, Cohen D and Berman H M 1992 Biopolymers 32725

32. Reddy C K, Das A and Jayram B 2001 J. Mol. Biol. 314 619

33. Cheng Y-K and Rossky P J 1998 Nature 392696

34. Cheng Y-K and Rossky P J 1999 Biopolymers 5742

35. Laage D and Hynes J T 2006 Science 311832

36. Chandra A 2000 Phys. Rev. Lett. 85768

37. Stillinger F H 1975 Adv. Chem. Phys. 311 